\title{
RECONSTRUÇÃO DO LIGAMENTO CRUZADO ANTERIOR COM DUPLO FEIXE UTILIZANDO OS TENDÕES DOS MÚSCULOS SEMITENDÍNEO E GRÁCIL: FIXAÇÃO COM DOIS PARAFUSOS DE INTERFERÊNCIA
}

\author{
ARTHROSCOPIC DOUBLE-BUNDLE RECONSTRUCTION OF ANTERIOR CRUCIATE LIGAMENT \\ USING HAMSTRING TENDON GRAFTS - FIXATION WITH TWO INTERFERENCE SCREWS
}

\begin{abstract}
Mario Carneiro', Ricardo Dizioli Navarro², Gilberto Yoshinobu Nakama ${ }^{3}$, João Mauricio Barretto4,
\end{abstract} Antonio Altenor Bessa de Queiroz ${ }^{5}$, Marcus Vinicius Malheiro Luzo ${ }^{6}$

\section{RESUMO}

Procedimentos cirúrgicos de reconstrução do ligamento cruzado anterior com duplo feixe dos tendões dos músculos semitendíneo e grácil têm sido descritos na última década. A maioria das técnicas descritas utiliza o dobro de material de síntese empregado na reconstrução com feixe único. Relatamos uma técnica original para a reconstrução do ligamento cruzado anterior com duplo feixe, na qual mantemos as inserções tibiais dos tendões dos músculos semitendíneo e grácil e realizamos dois túneis tibiais e dois túneis femorais. Os túneis femorais são realizados "de fora para dentro" e a fixação do enxerto é realizada somente com dois parafusos de interferência.

Descritores - Ligamento cruzado anterior; Traumatismos do joelho; Joelho

\begin{abstract}
Surgical procedures for double-bundle reconstruction of anterior cruciate ligament, which currently use semitendinous and gracilis tendon grafts, have been described in the last decade. Most of the techniques utilize twice the hardware used in single-bundle reconstructions. We report an original anterior cruciate ligament double-bundle reconstruction technique using semitendinous and gracilis tendon grafts, maintaining their tibial bone insertions with two tibial and two femoral tunnels. A simplified and precise outside-in femoral drilling technique is utilized, and the graft fixation is made utilizing only two interference screws.
\end{abstract}

Keywords - Anterior cruciate ligament; Knee injuries; Knee

uma taxa de pivot-shift residual de $15 \%$ na literatura ${ }^{(1)}$. Com o melhor conhecimento da anatomia e da função do LCA, diversas técnicas cirúrgicas de reconstrução têm sido desenvolvidas. A reconstrução do LCA com duplo feixe é atraente porque procura reproduzir a anatomia e função do LCA.

Desde 1999, vários cirurgiões têm realizado reconstruções anatômicas do LCA com duplo feixe ${ }^{(2,3)}$. A localização da origem femoral do feixe posterolateral(PL) tem sido um tema de debate. A localização do feixe PL

\footnotetext{
1 - Professor Afiliado-Doutor do Departamento de Ortopedia e Traumatologia da Unifesp-EPM; Vice-Chefe da Disciplina de Ortopedia do DOT- Unifesp-EPM, Brasil.

2 - Livre-Docente do Departamento de Ortopedia e Traumatologia da Unifesp-EPM, Brasil.

3 - Médico do Grupo do Joelho do Departamento de Ortopedia e Traumatologia da Unifesp-EPM, Brasil.

4 - Doutor; Chefe do Serviço de Ortopedia e Traumatologia da Santa Casa do Rio de Janeiro, Brasil.

5 - Mestre; Chefe Interino do Grupo do Joelho do Departamento de Ortopedia e Traumatologia da Unifesp-EPM, Brasil.

6 - Doutor; Médico do Grupo do Joelho do Departamento de Ortopedia e Traumatologia da Unifesp-EPM, Brasil.
} 
(com o joelho em $90^{\circ}$ de flexão) é distal e posterior ao feixe anteromedial $(\mathrm{AM})^{(3-6)}$. Do ponto de vista biomecânico, estudos recentes têm mostrado que a reconstrução do LCA com duplo feixe pode melhorar a cinemática normal do joelho ${ }^{(7)}$. Essas conclusões são apoiadas por estudos clínicos prospectivos, que mostram a redução da gaveta anterior e melhor controle da rotação na reconstrução do LCA com duplo feixe, quando comparada com a reconstrução com feixe único ${ }^{(4)}$. A grande maioria dos procedimentos cirúrgicos com duplo feixe utiliza o enxerto dos tendões dos músculos semitendíneo e grácil (STG). Todos os procedimentos, entretanto, utilizam o dobro do material de síntese para a fixação do enxerto em relação à reconstrução do LCA com o feixe único. O objetivo desta técnica é reconstruir o ligamento cruzado anterior com duplo feixe utilizando o enxerto dos tendões dos músculos semitendíneo e grácil, fixando-o com apenas dois parafusos de interferência, tal qual se faz na reconstrução do LCA com feixe único.

\section{TÉCNICA}

Uma incisão de cerca de 4cm de comprimento longitudinal é feita na porção anteromedial proximal da tíbia. Dissecamos os tendões dos músculos semitendíneo e grácil mantendo as inserções tibiais (Figura 1). Utilizando a técnica artroscópica, perfuramos os túneis tibiais anteromedial (AM) e posterolateral (PL). Primeiramente, realizamos o túnel tibial AM da maneira usual com a saída na região anteromedial da inserção tibial do LCA. Esse túnel tem orientação mais sagital $\left(20^{\circ}\right.$ do plano sagital e a $50^{\circ}$ do plano horizontal). O túnel tibial PL se origina anteriormente à inserção tibial do ligamento colateral medial superficial e termina no aspecto

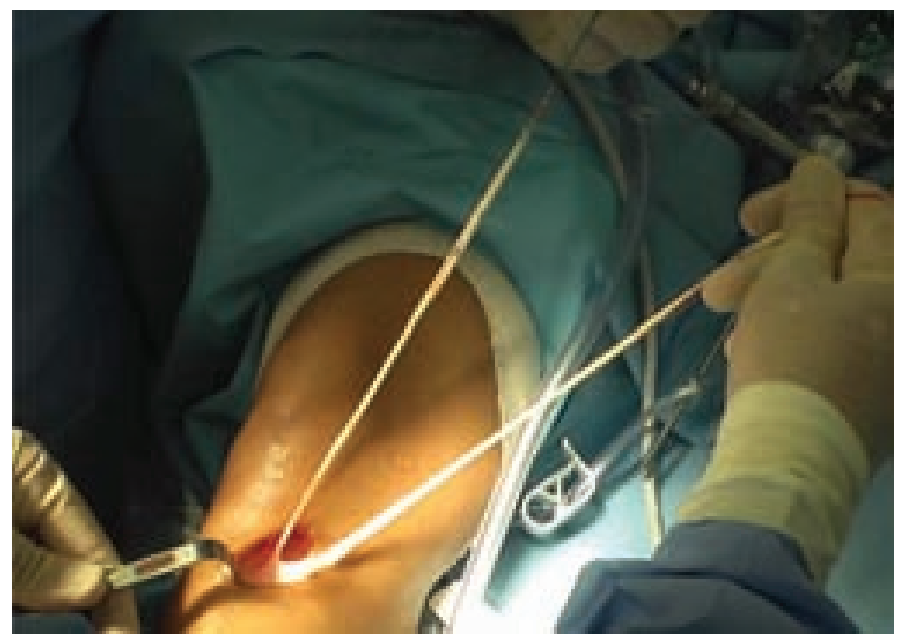

Figura 1 - Tendões flexores dos músculos semitendíneo e grácil mantendo as inserções tibiais posterolateral da inserção tibial do LCA. Esse túnel se situa num plano mais coronal $\left(45^{\mathrm{O}}\right.$ para o plano horizontal e sagital). Ambos os túneis tibiais são perfurados com uma broca de 6mm (Figuras 2 e 3). Em seguida, confeccionamos os túneis femorais AM e PL de fora para dentro com o auxílio de um guia femoral adequado. Posicionamos o fio-guia femoral AM 5mm anterior à cortical posterior do côndilo femoral lateral, às $9 \mathrm{~h}$ no joelho direito ou às 3 h no joelho esquerdo (Figura 4). $\mathrm{O}$ fio-guia femoral PL é posicionado $6 \mathrm{~mm}$ distalmente e $30^{\circ}$ posteriormente ao fio-guia femoral AM (Figura 5). Os túneis femorais são então perfurados com uma broca de $6 \mathrm{~mm}$ de fora para dentro (Figuras 2 e 6).

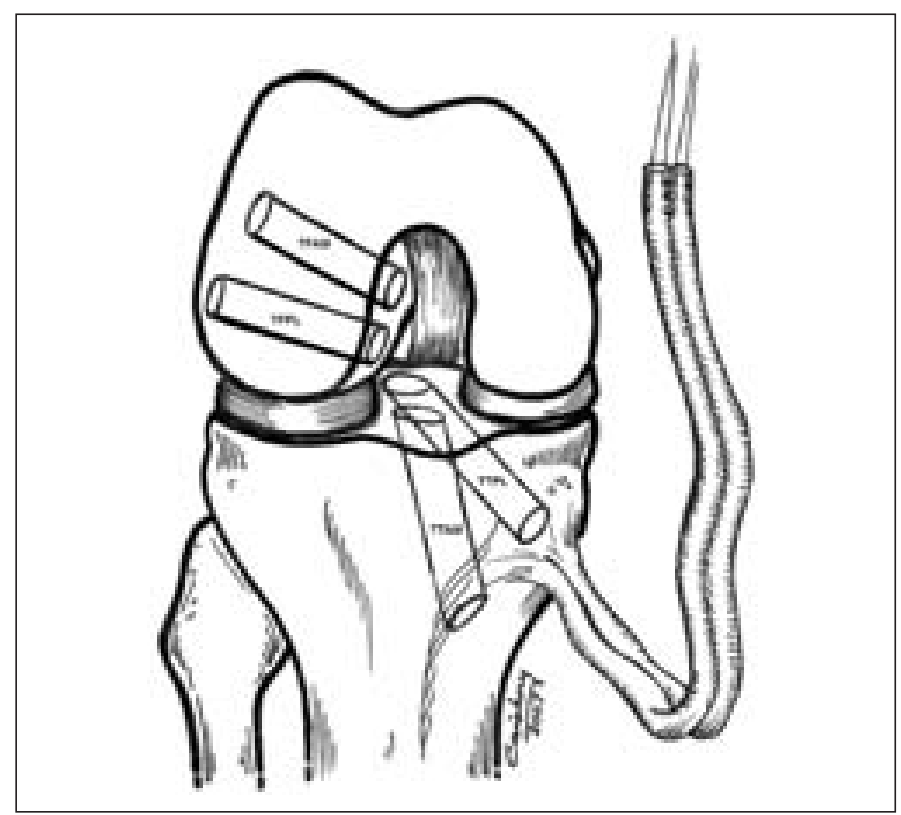

Figura 2 - Os tendões flexores são dissecados e suas inserções tibiais são mantidas. TTAM - túnel tibial anteromedial. TTPL túnel tibial posterolateral. TFAM - túnel femoral anteromedial. TFPL - túnel femoral posterolateral

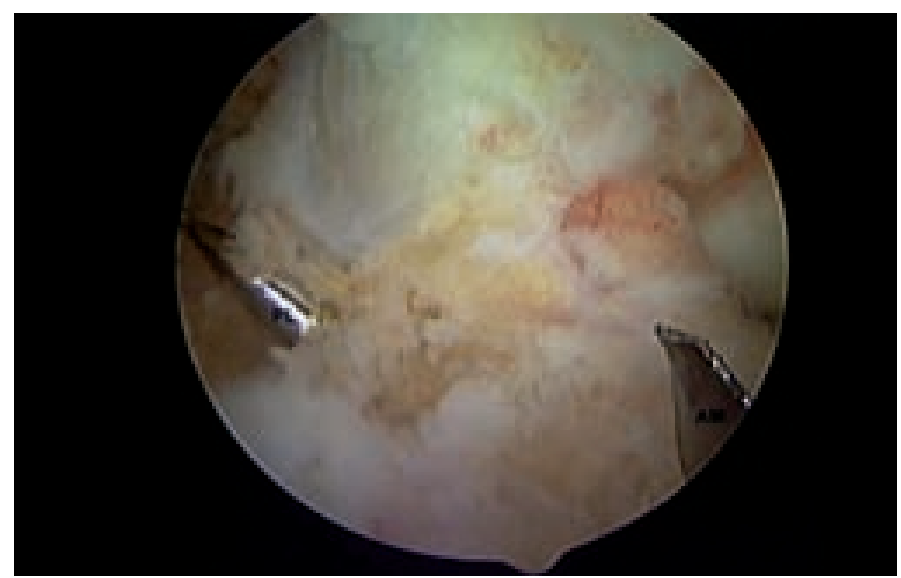

Figura 3 - Visão artroscópica dos fios-guia correspondentes aos túneis tibiais anteromedial $(\mathrm{AM})$ e posterolateral (PL) 


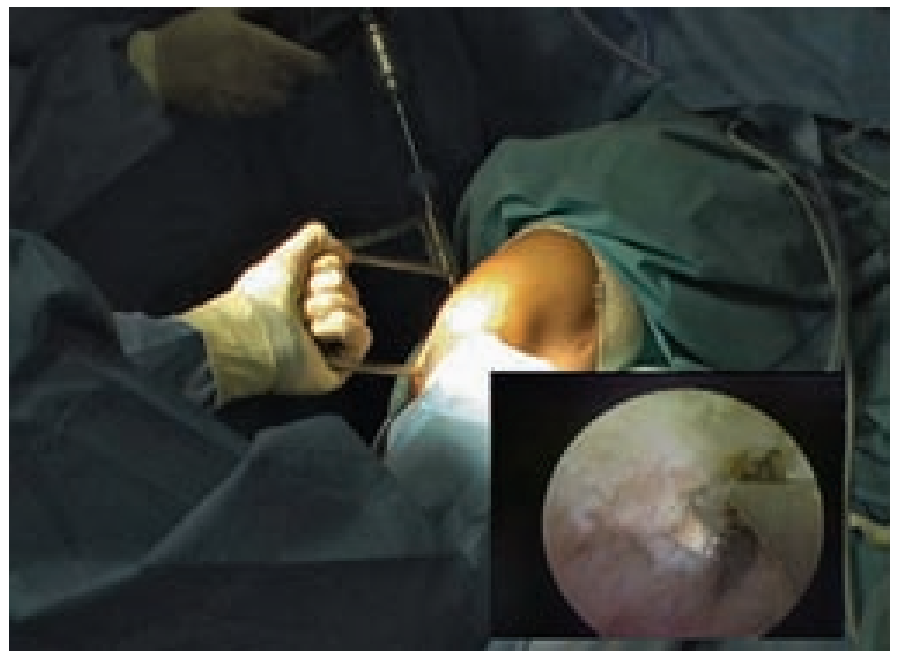

Figura 4 - Passagem e posicionamento do fio-guia para a confecção do túnel femoral AM. Visão artroscópica em detalhe: fio às $9 \mathrm{~h}$ - joelho direito

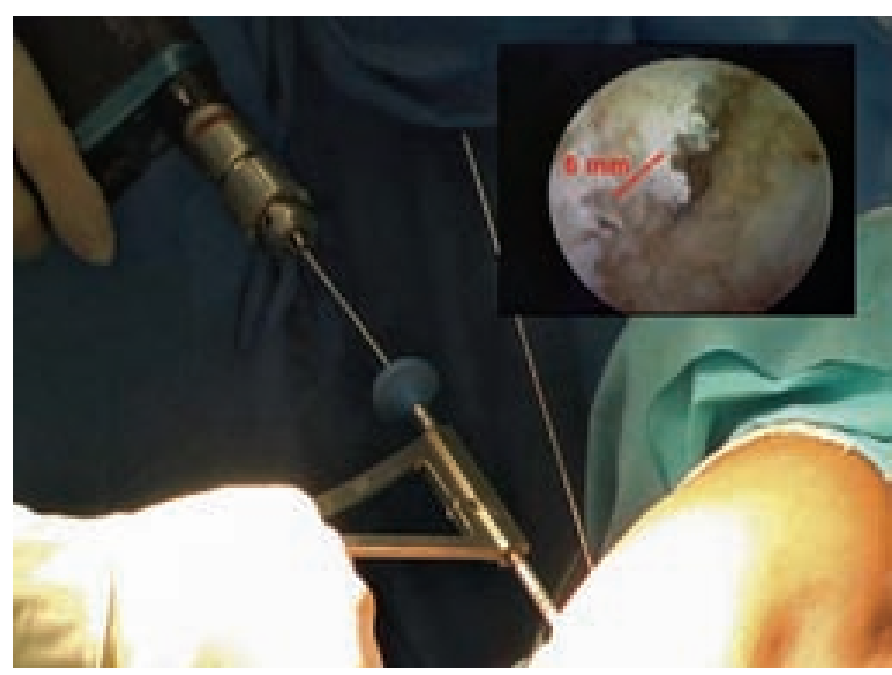

Figura 5 - Passagem do fio-guia para a realização do túnel femoral posterolateral com detalhe artroscópico do posicionamento intra-articular de ambos os fios

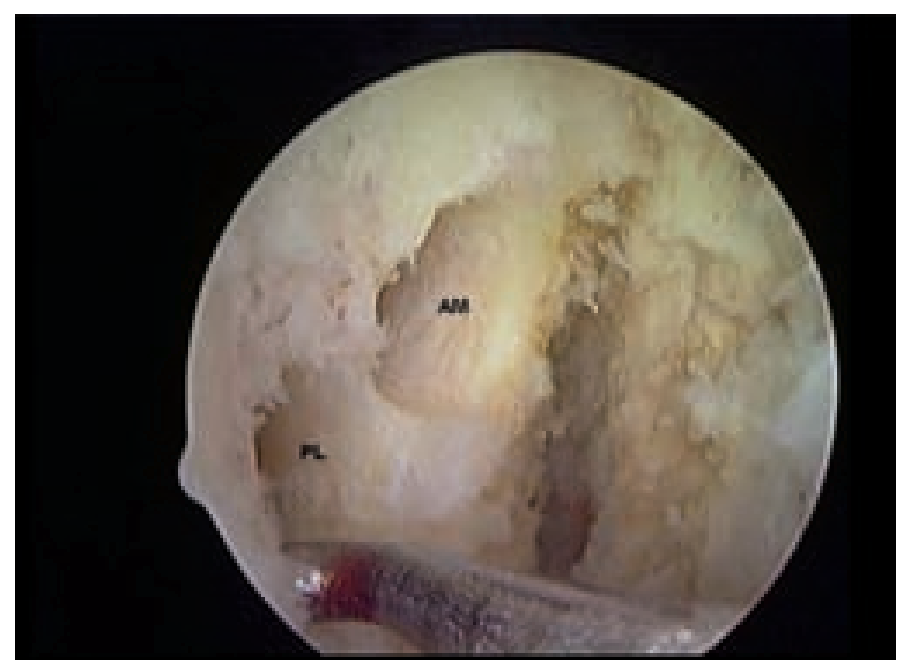

Figura 6 - Túneis femorais - visão artroscópica
Com o auxílio de um passador de tendão o enxerto é introduzido na articulação através do túnel tibial PL e exteriorizado na região lateral do joelho através do túnel femoral PL. O enxerto é tensionado e fixado de fora para dentro com um parafuso de interferência (metálico ou bioabsorvível, para partes moles) no túnel femoral posterolateral com o joelho em extensão de $15^{\circ}$ (Figuras 7 e 8). O restante do enxerto é reintroduzido na articulação pelo túnel femoral AM, sendo exteriorizado através do túnel tibial AM. Finalizando, o enxerto é tensionado e fixado com o joelho em flexão de $50^{\circ}$ (Figuras 9, 10 e 11). As pequenas incisões femoral e tibial são fechadas por planos.

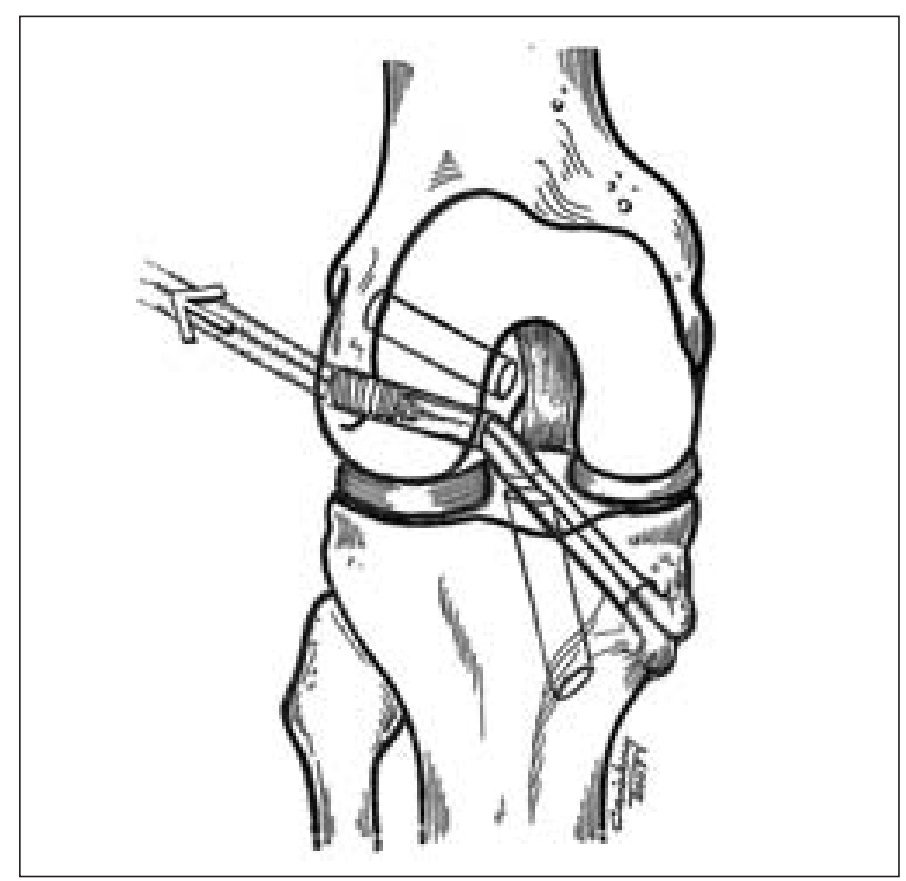

Figura 7 - Fixação femoral de fora para dentro do feixe posterolateral com o joelho em $15^{\circ}$ de extensão

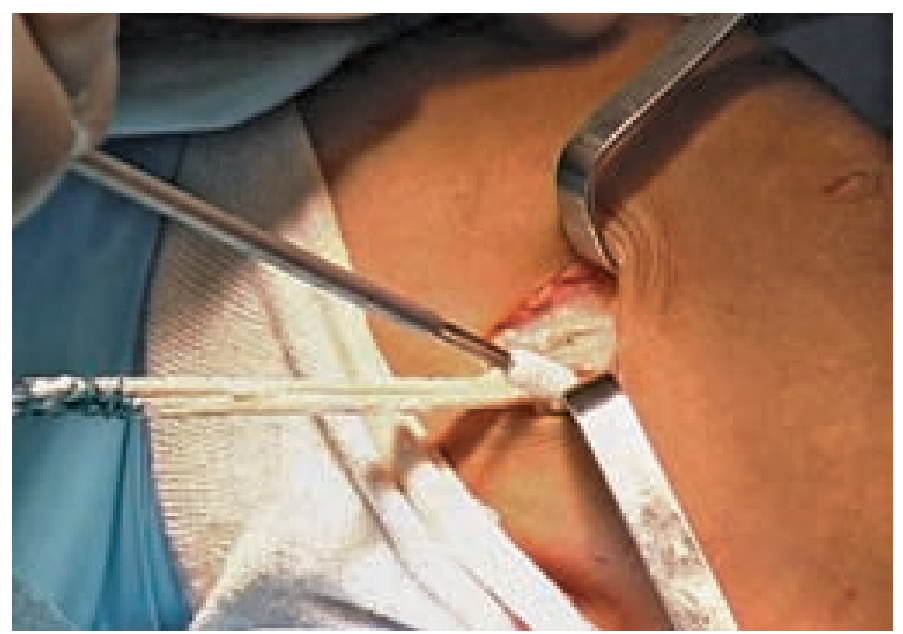

Figura 8 - Fixação do feixe posterolateral 


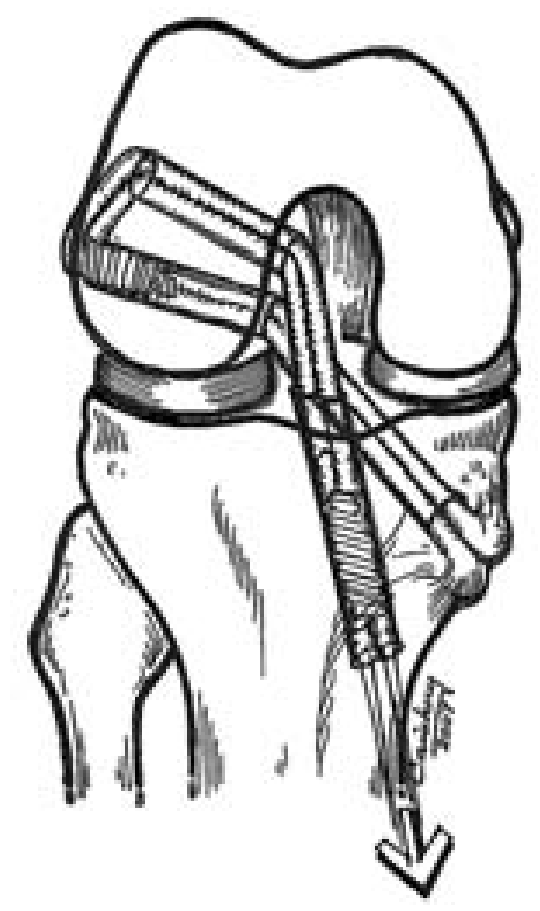

Figura 9 - Fixação do feixe anteromedial com o joelho em $50^{\circ}$ de flexão

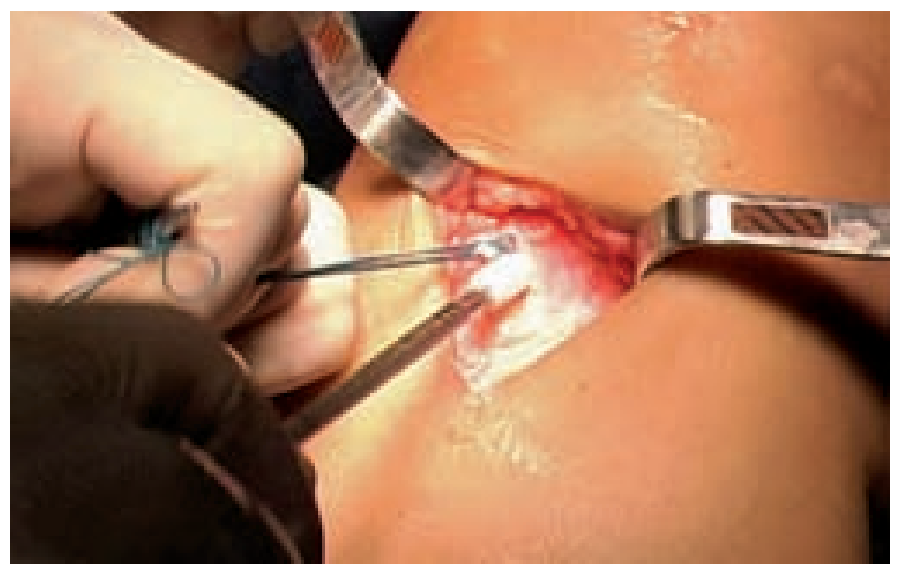

Figura 10 - Fixação do feixe anteromedial

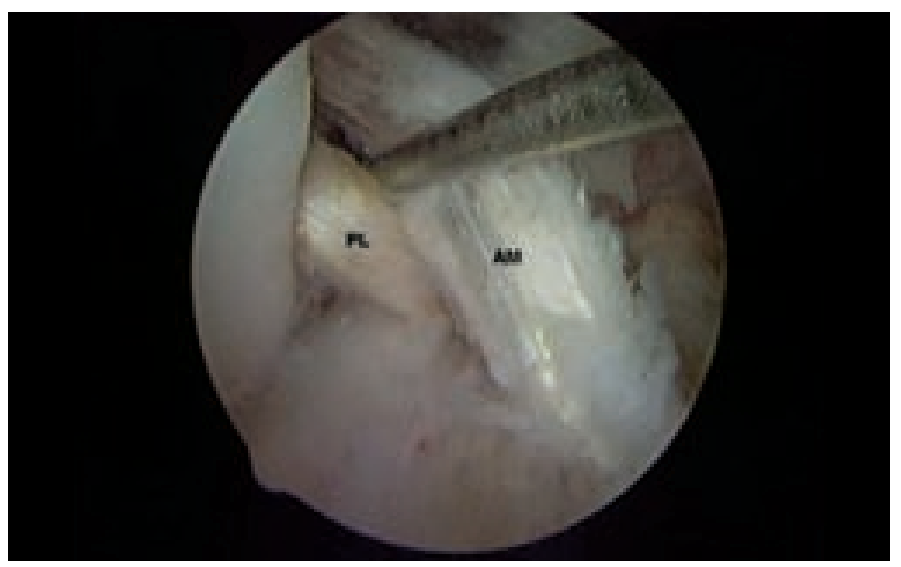

Figura 11 - Feixe AM tenso em flexão e PL em extensão
O pós-operatório é semelhante ao utilizado na reconstrução do LCA com feixe único.

\section{DISCUSSÃO}

O enxerto autólogo de tendões dos músculos semitendíneo e grácil é utilizado na maioria das técnicas de reconstrução do LCA com duplo feixe ${ }^{(2,8,9,10)}$. Há vários métodos de fixação do enxerto descritos na literatura: dois botões no fêmur e dois parafusos de interferência na tíbia, dois botões no fêmur e dois botões na tíbia, dois parafusos de interferência no fêmur e outros dois na tíbia, dois botões no fêmur e dois agrafes na tíbia e dois botões no fêmur e amarria em poste na tíbia ${ }^{(2,8-11)}$.

A técnica proposta por nós utiliza apenas dois parafusos de interferência, que é um material de síntese usual, de fácil obtenção e de baixo custo. Uma analise comparativa do custo do material de síntese utilizado na reconstrução do LCA com duplo feixe, considerando-se os diversos tipos de fixação disponíveis, é apresentada na Tabela 1 . Nessa tabela apresentamos o preço mínimo encontrado numa cotação do custo dos materiais de implante em três empresas de São Paulo. Acreditamos que o custo seja um fator importante a ser avaliado em nosso meio ao considerarmos que a reconstrução do LCA com duplo feixe possa apresentar melhores resultados em relação à reconstrução com feixe único. A nosso ver, não seria justo que fatores econômicos limitassem a utilização de uma técnica que visa melhorar ainda mais os resultados em nossos pacientes. Procuramos, então, solucionar esse problema desenvolvendo uma técnica anatômica, simples e de baixo custo, que poderá ser realizada pela maioria dos cirurgiões em nosso país.

Tabela 1 - Analise comparativa do custo do material de síntese nos diferentes métodos de fixação utilizados na reconstrução do LCA com duplo feixe

\begin{tabular}{c|c|c|c|c|c}
\hline Técnica & $\begin{array}{c}2 \text { botões femorais } \\
+2 \text { PIB na tíbia }\end{array}$ & 4 PIB & 4 PIM & 2 PIB & 2 PIM \\
\hline $\begin{array}{c}\text { Custo } \\
\text { (R\$) }\end{array}$ & $8.200,00$ & $6.400,00$ & $2.420,00$ & $3.200,00$ & $1.210,00$ \\
\hline
\end{tabular}

PIM - parafuso de interferência metálico.

PIB - parafuso de interferência bioabsorvível 
Consideramos ainda como outra vantagem dessa técnica a preservação da inserção tibial dos tendões do semitendíneo e grácil, o que causa menos lesão e confere maior viabilidade ao enxerto ${ }^{(12)}$.

Embora o objetivo deste manuscrito não seja a apresentação de resultados, podemos dizer que os primeiros 25 pacientes operados no período de julho de 2007 a março de 2009 vêm apresentando evolução bastante satisfatória. Uma análise comparativa dos resultados com outras técnicas está sendo realizada e esperamos poder emitir uma opinião mais consistente sobre esta técnica assim que tivermos maior casuística e maior tempo de seguimento.

\section{AGRADECIMENTO}

Agradecemos ao Prof. Dr. Caio Augusto de Souza Nery, Professor Associado do Departamento de Ortopedia e Traumatologia da Universidade Federal de São Paulo - Escola Paulista de Medicina, pelas ilustrações da técnica cirúrgica.

\section{REFERÊNCIAS}

1. Nedeff DD, Bach BR Jr. Arthroscopic anterior cruciate ligament reconstruction using patellar tendon autografts: A comprehensive review of contemporary literature. Am J Knee Surg. 2001;14(4):243-58.

2. Muneta T, Sekiya I, Yagishita K, Ogiuchi T, Yamamoto H, Shinomiya K. Twobundle reconstruction of the anterior cruciate ligament using semitendinosus tendon with endobuttons: operative technique and preliminary results. Arthroscopy. 1999;15(6):618-24.

3. Yasuda K, Kondo E, Ichiyama H, Kitamura N, Tanabe $Y$, Tohyama $H$, et al. Anatomic reconstruction of the anteromedial and posterolateral bundles of the anterior cruciate ligament using hamstring tendon grafts. Arthroscopy. 2004;20(10):1015-25.

4. Yasuda $\mathrm{K}$, Kondo E, Ichiyama $\mathrm{H}$, Tanabe $\mathrm{Y}$, Tohyama $\mathrm{H}$. Clinical evaluation of anatomic double-bundle anterior cruciate ligament reconstruction procedure using hamstring tendon grafts: comparisons among 3 different procedures. Arthroscopy. 2006;22(3):240-51.

5. Garofalo R, Mouhsine E, Chambat P, Siegrist O. Anatomic anterior cruciate ligament reconstruction: the two-incision technique. Knee Surg Sports Traumatol Arthrosc. 2006;14(1):510-6.

6. Mochizuki T, Muneta T, Nagase T, Shirasawa S, Akita K, Sekiya I. Cadaveric knee observation study for describing anatomic femoral tunnel place- ment for two-bundle anterior cruciate ligament reconstruction. Arthroscopy. 2006;22(4):356-61.

7. Gabriel MT, Wong EK, Woo SL, Yagi M, Debski RE. Distribution of in situ forces in the anterior cruciate ligament in response to rotatory loads. J Orthop Res. 2004;22(1):85-9.

8. Hamada M, Shino K, Horibe S, Mitsuoka T, Miyama T, Shiozaki Y, et al. Singleversus bi-socket anterior cruciate ligament reconstruction using autogenous multiple-stranded hamstring tendons with endoButton femoral fixation: A prospective study. Arthroscopy. 2001;17(8):801-7.

9. Takeuchi R, Saito T, Mituhashi S, Suzuki E, Yamada I, Koshino T. Double-bundle anatomic anterior cruciate ligament reconstruction using bone-hamstring-bone composite graft. Arthroscopy. 2002;18(5):550-5.

10. Zaricznyj B. Reconstruction of the anterior cruciate ligament of the knee using a doubled tendon graft. Clin Orthop Relat Res. 1987;(220):162-75.

11. Crawford C, Nyland J, Landes S, Jackson R, Chang HC, Nawab A, et al. Anatomic double bundle ACL reconstruction: a literature review. Knee Surg Sports Traumatol Arthrosc. 2007;15(8):946-64.

12. Kim SJ, Kim, HK, Lee YT. Arthroscopic anterior cruciate ligament reconstruction using autogenous hamstring tendon graft without detachment of the tibial insertion. Arthroscopy. 1997;13(5):656-60. 